\title{
1997 Von Hippel Award Goes to Gabor A. Somorjai for Broad Contributions to Surface Science
}

The Materials Research Society's highest honor, the Von Hippel Award, this year goes to Gabor A. Somorjai, professor of chemistry at the University of CaliforniaBerkeley. He is cited for "his extraordinary multidisciplinary contributions to the atomic-level understanding of materials surfaces and surface processes with technological importance in heterogeneous catalysis, corrosion, and tribology." Somorjai's ideas and his vision for the future as well as his promotion of the field and of his colleagues' work has had a major impact in stimulating support and raising the visibility of surface science when the field was young. The Von Hippel Award is given annually to an individual in recognition of outstanding contributions to interdisciplinary research on materials. Somorjai is also senior scientist and group leader of the Surface Science and Catalysis Program of the Center for Advanced Materials at the Lawrence Berkeley National Laboratory.

Somorjai has a wide interdisciplinary approach to research, and is known to have graduate students and postdoctorates from a broad range of disciplines working in his laboratory. This approach supports the much needed bridge between fundamental research and industrial applications. His frontier research is often guided by technological need, manifested by close collaboration with a variety of industrial research laboratories. His experimental and theoretical studies on molecular-scale structure and bonding at solid surfaces, and mechanisms of surface reactions have identified basic causes responsible for behavior at interfaces observed at the industrial level, and thereby helped develop new and better surface materials and surface technologies. Over $80 \mathrm{PhD}$ students and 120 postdoctorates have been guided by his creativity, experimental practicality, and unabashed curiosity.

The science of surfaces and catalysis as developed by Somorjai over the last 30 years has evolved techniques that are in widespread use today to explore the relationship between chemical bonding and heterogeneous catalysis. These tools of surface science are used throughout industry in tribology, bioengineering, microelectronics, automotive technology, and environmental sciences, among others. At Intel, his former students now lead development activities in plasma etching, polymer-metal adhesion for packaging, and surface analytical techniques.

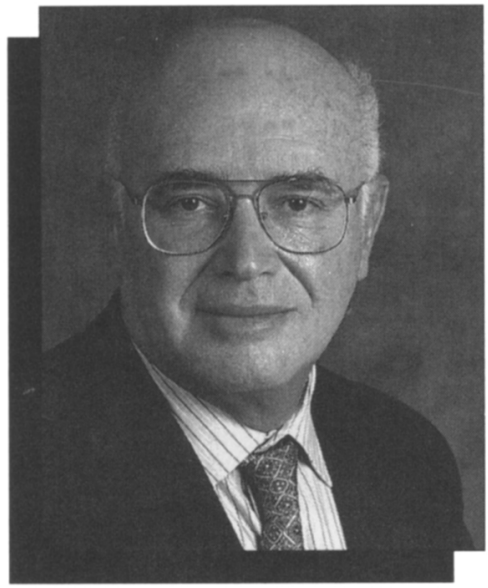

Gabor A. Somorjai

Somorjai has carried out a program to build a fundamental molecular basis for the surface science of heterogeneous catalysis. He characterized clean single-crystal surfaces, the structure and bonding of adsorbed molecules, and carried out catalytic reactions on these crystal surfaces. He discovered the relationship between the atomic structure of surface sites and their catalytic activity, and his research has uncovered many new concepts for adsorbed monolayer chemical bonding and heterogeneous catalysis.

Much of Somorjai's research has been aimed at elucidating the structure of metal surfaces, as well as adsorbed monolayers of molecules on metals. He contributed greatly to the development of low-energy electron diffraction (LEED) surface crystallography, and in 1965 reported that atomic sites on the (100) surface of platinum crystals were different from those in the bulk. In a subsequent series of experiments, he explained the properties of reconstructed metal surfaces and demonstrated that surface irregularities, such as steps and kinks, were largely responsible for the chemical activity of transition metals. His recent studies have reported the reconstruction of oxide surfaces.

Along with his characterization of clean metal surfaces, Somorjai explained the structures of adsorbed organic molecules, the chemical rearrangements of adsorbed ethylene and other alkenes and alkynes, and reported his discovery of alkylidyne bonding. His fundamental work using LEED and high-resolution electron energy loss spectroscopy made it possible to identify that the bonding of hydrocarbons is similar to that in organometallic clusters.
His studies of adsorbed ordered and disordered monolayer surface structure by LEED surface crystallography reveal coadsorption-induced ordering.

Somorjai has been a major contributor in developing new instrumentation for studies in structural surface chemistry and the surface science of heterogeneous catalysis. He developed several innovations in LEED instrumentation. An apparatus designed in his laboratory combined the high vacuum technology required for surface analysis with the high pressure cell necessary for studies of catalytic reactions on small area crystal surfaces.

The low pressure-high pressure technology was extended to incorporate a scanning tunneling microscope operating at high temperatures and pressures, which permitted in situ monitoring of reactioninduced restructuring of metal crystal surfaces. He also used sum frequency generation-vibrational spectroscopy to identify reaction intermediates that form during catalytic reactions. Thus, the surface technology developed by the Somorjai group evolved from molecular studies of surfaces before and after catalytic reactions to studies during the reaction. He was the first to develop a molecular beam-surface-scattering instrument to determine energy and angular distributions in single-crystal surface reactions. The combination of technical development and its application to significant problems of surface chemistry and heterogeneous catalysis is a main characteristic of Somorjai's research.

Somorjai's work has dealt mainly with investigation of catalytic reactions on single-crystal surfaces. He studied the conversion of hydrocarbons over platinum, demonstrating the surface structure sensitivity of reaction rates and product distribution, along with the key role that atomically rough surfaces, steps, and kinks play in controlling reactivity. The focus of these studies included ammonia synthesis over iron and rhenium single crystals, along with carbon monoxide and ethylene hydrogenation, and thiophene hydrodesulfurization over various transitionmetal crystal surfaces (Pt, Rh, Mo, Re, Fe).

The new concepts of heterogeneous catalysis uncovered as a result of these studies include the importance of atomically rough surfaces for carrying out high rate surface reactions, the presence of a strongly bound overlayer on the active catalyst surface (carbonaceous or sulfur-containing), weakly bound reaction intermediates, the unique high activity of bimetal- 
lic systems and certain oxide-metal interfaces, and the crucial role that coadsorbed "promoters" play (such as potassium, alumina, and sulfur) as either structure or bonding modifiers. Adsorbate-induced restructuring of catalysis surfaces, occurring on different time scales (adsorption, catalytic turnover, or longer times), has been suggested as an important event that controls catalytic site activity.

This work has led to the exciting possibility of utilizing the molecular ingredients of catalysis to design new catalysts with tailored activity and selectivity. The Somorjai group has shown that complex, multicomponent catalysts can be constructed using single-crystal surfaces with the proper surface structure as substrates.
Somorjai serves on the editorial boards of 12 journals, has published over $700 \mathrm{sci}-$ entific papers, and has written four books-Introduction to Surface Chemistry and Catalysis (1994), Chemistry in Two Dimensions: Surfaces (1981), Monolayers on Solid Surfaces (1979), and Principles of Surface Chemistry (1972). He has been awarded honorary degrees by universities in Brussels, Paris, and Budapest, and among many other awards and honors, he is a member of the National Academy of Sciences and the American Academy of Arts and Sciences, an honorary member of the Hungarian Academy of Sciences, and a fellow of the American Association for the Advancement of Science and the American Physical Society.
Born in Budapest, Hungary, Somorjai was a fourth-year student in chemical engineering at the Technical University in Budapest when the Hungarian revolution broke out in 1956. He moved to the United States, and in 1960 received a $\mathrm{PhD}$ degree in chemistry from UC-Berkeley. He was on the research staff at IBM in Yorktown Heights, New York for the first four years of his career, and in 1964 joined the Department of Chemistry at Berkeley, becoming a full professor in 1972 .

Somorjai will present his talk, "From Surface Materials to Surface Technologies," at the Awards Ceremony of the MRS Meeting in Boston, Massachusetts, on Wednesday, December 3 at 6:00 p.m. in Salon E, Marriott Hotel.

\section{Merton C. Flemings Selected for 1997 David Turnbull Lectureship for Contributions to Solidification Processing}

Merton C. Flemings, Toyota Professor of Materials Processing at the Massachusetts Institute of Technology, has been selected as the 1997 recipient of the David Turnbull Lectureship. He was cited for "his contributions to the foundations and technology of solidification processing and for educating a generation of materials engineers." The David Turnbull Lectureship recognizes the career of a scientist who has made outstanding contributions to understanding materials phenomena and properties through research, writing, and lecturing, as exemplified by David Turnbull.

Flemings's research over the last 40 years has spanned both the basic and applied science of solidification processing, and has had broad impact on solidification theory and experiments, as well as on industrial practices. Even today, his 1974 text, Solidification Processing, is a widely used teaching and reference book for students and practitioners. The paper he and his students published in the early 1960s on microsegregation later became the most extensively referenced paper in the Science Citation Index. He later broadened this work to quantitative analysis of macrosegregation, which radically altered the thencurrent understanding of the topic. His theoretical analyses are now widely used for prediction and control of macrosegregation in continuous casting.

Flemings showed that eutectic-like structures could be obtained in two-phase alloys far from eutectic composition, and he established the basic principles for directional solidification of these in situ composites. He and his students discovered and quantified the basic mechanism of establishing dendrite arm spacings and casting in ingots ("coarsening"), and later

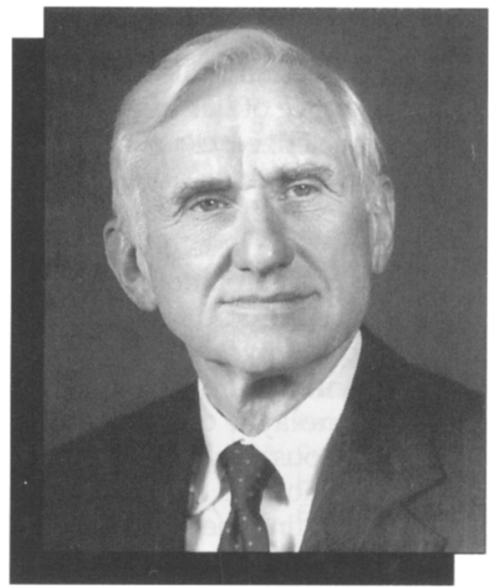

Merton C. Flemings

showed that dendritic structures could be fully eliminated by vigorous agitation during solidification. An industry for forming "semi-solids" developed from this work. He was also the first to show that a dc magnetic field acts as an effective "brake" on fluid flow in solidification processing of metals.

He described mathematically and defined experimentally the fundamentals of flow and solidification behavior in metal matrix composites. His work on the solidification mechanism YBCO superconductors now forms the foundation for, and describes the limits of, practical directional solidification processes for these materials.

This research has led to numerous practical applications, including "premium quality" aluminum castings, "foam vaporization" casting of automotive engine components, braking of fluid flow in con tinuous casting by application of a de magnetic field, semi-solid casting, and reactive melting of intermetallic alloys. Both the foam vaporization process and semi-solid forming process are now used to produce millions of automotive parts each year.

Flemings became head of the Department of Materials Science and Engineering at the Massachusetts Institute of Technology (MIT) in 1982, a position he held until 1995. During this period, he broadened what was effectively a metallurgy department with a small ceramics program to a department with strength across all classes of materials.

After receiving his $\mathrm{ScD}$ degree from $\mathrm{MIT}$ in 1954, Flemings worked in private industry for two years before joining the MIT faculty. He co-chaired the National Materials Study for the National Academy of Sciences in the late 1980s, and served as a co-editor of the recently published Encyclopedia of Advanced Materials. He is the author or co-author of close to 300 papers, holds 26 patents, and has been honored by the Minerals, Metals \& Materials Society, ASM International, AIME, and industry and professional groups in France, Italy, and Japan. Flemings has been named the 1997 recipient of the Acta Metallurgica J. Herbert Hollomon Award. He is a member of the National Academy of Engineering and the American Academy of Arts and Sciences. He recently completed a term as a councillor of MRS.

Flemings will deliver the Turnbull Lecture, "Solidification Science and Engineering Practice," for Symposium B of the MRS Meeting in Boston, Massachusetts, on Mnnday, December 1, at 10:00 a m. in Amencan South, Westin Hutel. 